\title{
CHEMICAL EVOLUTION OF THE GALACTIC DISK AND BULGE
}

\author{
ROSEMARY F.G. WYSE \\ Department of Physics and Astronomy, The Johns Hopkins \\ University, Baltimore, MD 21218, USA (permanent address) \\ and \\ Center for Particle Astrophysics, University of California, \\ Berkeley, CA 94720, USA \\ (wyse@wyser.pha.jhu.edu)
}

\section{Introduction}

The Milky Way Galaxy offers a unique opportunity for testing theories of galaxy formation and evolution. The study of the spatial distribution, kinematics and chemical abundances of stars in the Milky Way Galaxy allows one to address specific questions pertinent to this meeting such as

(i) When was the Galaxy assembled? Is this an ongoing process? What was the merging history of the Milky Way?

(ii) When did star formation occur in what is now 'The Milky Way Galaxy'? Where did the star formation occur then? What was the stellar Initial Mass Function?

(iii) How much dissipation of energy was there before and during the formation of the different stellar components of the Galaxy?

(iv) What are the relationships among the different stellar components of the Galaxy?

(v) Was angular momentum conserved during formation of the disk(s) of the Galaxy?

(vi) What is the shape of the dark halo?

(vii) Is there dissipative (disk) dark matter?

Chemical evolution allows one to focus on particular aspects of galaxy formation, such as the stellar initial mass function (IMF), the star formation history - including the homogeneity of the star formation process and the rate of mass assembly to form the 'final' galaxy. Elemental abun- 
dance ratios play an important role in reaching fairly model-independent conclusions from the confrontation of theory with observations. The scatter about mean trends also can not be ignored, as was the common practice in the (recent) past, and understanding the physics of intrinsic variations in the star formation process will be a major step forward.

\section{Galaxy Formation}

I shall briefly summarise current ideas of galaxy formation and evolution to place Galactic work in context (see e.g. Silk and Wyse, 1993 for a more complete review, and White this volume). The nature of dynamically-dominant Dark Matter determines the way in which structure forms in the Universe. All density fluctuations are initially just gas and dark stuff, and the rate at which stars form is very model-dependent, but feedback from stars plays a major role in the energy balance. Hierarchical-clustering theories such as Cold Dark Matter (CDM; e.g. Blumenthal et al., 1984) predict that the first objects to collapse under self-gravity are a small fraction of the mass of a typical galaxy, so that galaxies form by merging of these smaller objects. The rate of merging and growth of mass of a protogalaxy can be estimated by studying the dark matter, which is assumed to be dissipationless and hence to have simpler physics than the baryonic component (but note that this provides an over-estimate of the merging rate of the dissipated, baryonic component e.g. Navarro, Frenk and White 1994). Lacey and Cole (1993) have a particularly vivid schematic representation of the merging process as a tree (their Figure 6), where time increases from top to bottom, and the width of the branches indicates the mass of a particular halo associated with galactic substructure. The final product, the galaxy, is represented by the single trunk at the ground, and the relative thicknesses of branches meeting reflect the mass ratios in a merger. The merging history of a galaxy is then described by the shape of the tree. The extremes of morphological type may perhaps be the result of merging histories that are described by the two types of trees that I at least was taught to draw as a child - either one main trunk from top to bottom, with many small branches joining the trunk at all heights, as a fir tree, or a main trunk that splits into two repeatedly, more like an English oak tree. The latter, dominated by 'major mergers' or equal mass mergers, may lead to an elliptical galaxy. The former, where the merging history is dominated by 'minor mergers', or very unequal mass mergers with a well-defined central core at all times, may lead to a disk galaxy. This picture of disk galaxy formation - building up by accretion of substructure onto a central core - provides a synthesis of elements of the much-discussed and previously apparently mutually-exclusive 'monolithic collapse' paradigm of Eggen, Lynden-Bell and Sandage (1962) 
and the 'chaotic' halo formation envisaged by Searle and Zinn (1978). One of the reasons for the popularity of CDM is its analytic tractibility; Gunn (1987) presents an elegant exposition of the formation of the Milky Way. Constraining the merging history - and future - of the Milky Way is of obvious importance, as is determination of the star formation history.

Early star formation in the proto-Galaxy is likely to occur not only in the central core, but also in low-mass substructure - perhaps of the mass of dwarf galaxies - in the envelope of the density profile surrounding the local peak in the (smoothed) density fluctuation field that is destined to become a galaxy. The onset of local gravitational instability and star formation in Galactic substructure will not be simultaneous but will reflect the natural spread in local density fluctuation amplitude (e.g. Silk and Wyse, 1993). The shallow depth of the potential well of these low-mass systems will most probably mean that their chemical evolution is truncated, with their first generation of stars capable of producing drastic effects such as supernovadriven winds (e.g. Dekel and Silk, 1986). The stellar halo probably formed from the merger of substructure like this (e.g. Hartwick, 1976; Searle and Zinn, 1978); the ejected gas will likely flow towards the central regions, based on angular momentum arguments (Carney, 1990; Wyse and Gilmore, 1992) and also from elemental abundance considerations (discussed below). Further build-up of the 'bulge' results from dynamical friction operating on the stellar components of accreted/merged systems and by gas inflow driven by gravitational torques during the merging process. Dissipation, and settling to the symmetry plane, of the gas which was not intimately involved in earlier star formation provides a disk. Again, the angular momentum of the disk at the solar neigbourhood is substantially higher than that of the stellar halo, and the half-mass radius of the stellar halo is significantly less than that of the disk, arguing against significant pre-enrichment of protodisk gas by mixing with the products of stellar evolution in the halo; this would suggest that the oldest disk stars - most probably now in the thick disk - should be metal-poor.

Early theories of disk-galaxy formation within dynamically-dominant dark-matter haloes assumed a fixed angular-momentum vector and detailed angular-momentum conservation (Fall and Efstathiou, 1980). More recent calculations have addressed the effects, within tidal-torque theory for the generation of angular momentum, of the inherent run of density fluctuations about a peak (e.g Ryden, 1988; Quinn and Binney, 1992). The spins of shells of material located at distances $r_{i}$ from the peak's location tend to be anti-correlated for $r_{1} / r_{2} \gtrsim 2$ (Quinn and Binney, 1992). Further, gas viscosity and dynamical friction will cause angular momentum re-distribution (e.g. Zurek, Quinn and Salmon, 1990; Katz and Gunn, 1991), augmented by torques due to non-axisymmetric perturbations such as a bar (e.g. Blitz and 
Spergel, 1991). The net effect is that not only will infall to the proto-disk be lumpy, due to the underlying spectrum of density fluctuations, but its angular momentum vector will be time-dependent, with resulting complicated final equilibrium. The disk can certainly not be treated as a 'closed-box' in these theories, but rather mass is added on long timescales, both as gas and as dissipationless material, namely the stars and dark matter of the halo substructure/dwarf galaxy satellites. The recent discovery of a satellite galaxy apparently in the process of being accreted by the Milky Way (Ibata, Gilmore and Irwin, 1994) is a graphic illustration of this process.

Since the pioneering work of Toomre (e.g., 1977) much effort has gone into study of the effects of both stellar and gaseous mergers upon galaxy morphology. Provided all the orbital energy of an accreted satellite, mass $M_{s a t}$, is available to increase the random energies of the stars in a thin disk of mass $M_{\text {disk }}$, then after a merger the thin disk will be heated by an amount (Ostriker, 1990)

$$
\Delta v_{\text {random }}^{2}=v_{\text {orbit }}^{2} M_{\text {sat }} / M_{\text {disk }} .
$$

Of course the internal degrees of freedom of the satellite could also be excited and any gas present could, after being heated, cool by radiation, so this is a definite upper limit to the heating of the disk. Note although there is indeed an age-velocity dispersion relationship for stars in the thin disk, the vertical velocity dispersion saturates at $\sim 20 \mathrm{~km} / \mathrm{s}$ for stars older than a few Gyr (e.g. Freeman, 1991). This may be understood if the source of the heating is confined to the thin disk itself, such as encounters with giant molecular clouds (e.g. reviewed by Lacey, 1991); some other mechanism is required for the thick disk, which has $\sigma_{Z} \sim 45 \mathrm{~km} / \mathrm{s}$.

The thick disk of the Milky Way Galaxy at least morphologically could be a remnant of the last significant (in terms of mass ratio) minor-merger event in the Galaxy's history (see e.g. Gilmore, Wyse and Kuijken, 1989). The most detailed N-body simulation of this process published to date (Quinn, Hernquist and Fullager, 1993) follows the accretion, by a disk galaxy, of a satellite galaxy with $10 \%$ of the mass of the disk. There are many parameters that need to specified, relating to the initial orbit and to the internal density profile and kinematics of the satellite, and of the disk. The generic evolution is that the satellite's vertical motion is damped rather quickly, with a slower radial decay to the central regions (displayed very clearly in their Figure 11). Both the radial and vertical structures of the disk are altered; the vertical heating increases the disk scale-height by about a factor of two. However, the orbital energy is indeed deposited both in the disk particles and in the internal degrees of freedom of the satellite; the final galaxy has a thick disk which consists of both heated thin-disk stars and shredded-satellite stars. The mix of these obviously depends on 
the (many) model parameters, both orbital and internal to the galaxies. One may expect the chemical evolution of the disk and of the satellite to have been different; this should be observable in the chemical abundances of thick-disk stars.

\section{Chemical Evolution - The Importance of Element Ratios and of Scatter}

Chemical evolution models should incorporate the inherent spatial and temporal inhomogeneity and gas flows of realistic galaxy formation models but avoid getting lost in parameter space. Elemental abundances provide a means to break the degeneracy found when simply overall 'metallicity' is followed - the lack of uniqueness of model fits to observations of 'metallicity' is well documented (e.g. Tosi, 1988). And one should remember that the fact that a model 'fits' a particular observation is not a proof of its appropriateness or its correctness, but rather that one should move to the next level of prediction/comparison with observation. The overwhelming majority of published models for the chemical evolution of the Galaxy are concerned only with fitting the mean trend with time, assuming a one-toone correlation between time and metallicity. This is due in part to the large observational uncertainties of earlier datasets (see Tinsley, 1975, who neverthe-less investigated the possible influence of a spread in metallicity on the G-dwarf problem). However, as discussed by Edvardsson et al. (1993) and by Nissen (this volume), real intrinsic scatter has now been unambiguously detected in the age-metallicity relation for solar neighbourhood samples of F/G stars, with scatter of a few Gyr at given metallicity, or a few tenths of a dex at given age. Indeed, one could plausibly argue that the scatter is as large as any mean trend. This scatter has to be taken into account in models and provides more stringent constraints on theories. Star formation should be modelled as a local process, within molecular cloud complexes, forming stellar associations with internal enrichment prior to disruption ( $c f$. Tinsley, 1976) and possible re-formation (Gilmore and Wyse, in prep.).

Element ratios, by contrast, show little intrinsic scatter (e.g. Edvardsson et al., 1993; Nissen, this volume). Element ratios are very important for understanding galactic evolution, in part since different elements come from different sources, and are ejected into the interstellar medium on different timescales (Tinsley, 1979). For example, oxygen is predominantly synthe-

sized in short-lived, massive stars that explode in Type II supernovae, while iron has an additional important source in Type Ia supernovae. The best, but by no means generally accepted, current model for these latter supernovae invokes the eventual merging, driven by gravitational radiation, of a pair of $\mathrm{CO}$ white dwarfs, with total mass greater than the Chandrasekhar 
mass (Iben and Tutukov, 1987). Thus the explosion timescale is set not by intrinsic stellar parameters, but rather by orbital parameters, and can be a Hubble time (note that this is a problem for advocates of white dwarfs as the dark matter in the haloes of galaxies, unless one suppresses the binary fraction of the progenitor stars by several orders of magnitude below that of even the old, metal-poor stars in our Galaxy; Smecker and Wyse, 1991). Detailed models suggest that of order half of the possible progenitors (white dwarf binaries of suitable orbits and masses) from a single burst of star formation explode in $\sim 10^{9} \mathrm{yr}$ (e.g. Smecker-Hane and Wyse, 1992). The ratio of oxygen-to-iron then is determined in large part by the ratio of present-to-past star formation rates.

Current understanding of supernova rates and nucleosynthesis implies that for a solar neighbourhood IMF, Type Ia and Type II supernovae each provide a yield of somewhat below solar iron ( $c f$. Rana, 1991). Thus a stellar population whose mean iron abundance is significantly above solar either self-enriched sufficiently slowly that Type Ia supernovae contributed, or had an IMF rather biased towards massive stars. This latter possibility will be detectable in the value of the element ratios. The dependence on IMF arises since the mass of oxygen ejected in a Type II supernova is a steeply increasing function of progenitor mass, while iron, if anything, decreases with progenitor mass (see Wyse and Gilmore, 1992). The uncertainties in supernova nucleosynthesis preclude a robust prediction of the $[\mathrm{O} / \mathrm{Fe}]$ ratio typical from a generation of massive stars, but offsets as one varies the massive-star IMF away from that of the solar-neighbourhod can be made with some confidence. The simplest analysis is done for a power-law IMF. An IMF biased towards massive stars by a change of slope of order unity in the power-law index will result in an enhanced $[\mathrm{O} / \mathrm{Fe}]$ of about 0.3 above that value - itself about 0.3 , or a factor of two above the solar ratio - for a solar neighbourhood massive-star IMF.

To illustrate the utility of elemental abundances, consider three independent star-forming regions, each with its own star formation history and IMF. The pattern of $[\mathrm{O} / \mathrm{Fe}]$ versus $[\mathrm{Fe} / \mathrm{H}]$ expected in stars formed therein is shown schematically in Figure 1. Generically, one predicts a plateau at early stages, when Type II supernovae dominate the chemical enrichment, followed by a downturn to lower values of $[\mathrm{O} / \mathrm{Fe}]$ after the elapsed time is long enough for Type I supernovae to have occurred and their iron to be incorporated in the interstellar medium, and thus into subsequent generations of stars. It is clearly important to determine the iron abundance at which the downturn occurs, since this allows one to assign a (relative) age to this metallicity ( $c f$. Matteucci and Greggio, 1986; Truran, 1987; Wyse and Gilmore, 1988; Pagel, 1989a, 1994).

The dashed line in Figure 1 indicates the evolution of a region with an 
Figure 1. Schematic description of the $[\mathrm{O} / \mathrm{Fe}]$ element ratio evolution as a function of $[\mathrm{Fe} / \mathrm{H}]$ for three independent star-forming regions of differing massive-star IMFs and star formation rates.

IMF dominated by massive stars, compared to the other two, which have a massive star IMF similar to the population represented now by the halo subdwarfs. The biased IMF results in a higher enhancement of $[\mathrm{O} / \mathrm{Fe}]$ for the mean Type II supernova, while the higher nucleosynthetic yield of this region means that, for a given star-formation rate, a higher $[\mathrm{Fe} / \mathrm{H}]$ can be reached prior to the onset of Type Ia supernovae, which occurs at a fixed time after the initiation of star formation in that region. Of the remaining two regions, the solid line indicates the evolution with a higher starformation rate at early times, and so has more enrichment through successive generations of massive stars prior to the onset of chemically-detectable Type Ia supernovae. An important feature of chemical enrichment by Type I supernovae is that it can occur without accompanying star formation, since in most models, the time lag between the formation of the progenitor stars and the explosion is set by binary orbital parameters and can be very long. Thus a system where the star formation occurs in bursts, separated by periods of little or no star formation, can show very different patterns of element ratios compared to monotonic star formation (e.g. application to the Magellanic Clouds by Gilmore and Wyse, 1991). Such a possibility is indicated schematically by the upturn in the dot-dashed line in the Figure, which would happen if the star-formation rate abruptly increased signifi- 
cantly, providing many more Type II supernovae per Type I supernova. A true hiatus in star formation would be detectable in element ratios by a gap in oxygen-to-iron versus iron, since iron enrichment from Type I supernovae would continue during the hiatus and subsequent stars to form will have incorporated this 'extra' iron, without accompanying oxygen.

\section{Application to the Bulge}

The pattern of $[\mathrm{O} / \mathrm{Fe}]$ versus $[\mathrm{Fe} / \mathrm{H}]$ for stars observed at the solar neighbourhood is similar to the solid line in Figure 1 (e.g. Wheeler, Sneden and Truran, 1989). The two notable features of the data most relevant for this discussion are firstly that there is little detectable scatter about the mean trend, and secondly that the element ratios are correlated with kinematics - stars with halo kinematics have enhanced [O/Fe] (e.g. Barbuy and Erdelyi-Mendes, 1989, their Table 4). The hierarchical-merging picture for the formation of the Galaxy outlined above might lead one to expect a random mix of all the patterns indicated in Figure 1. That this does not occur indicates that the massive star IMF is constant in time and place (e.g. Gilmore and Wyse, 1993; Nissen et al., 1994) and further that chemical evolution in the halo substructure is truncated prior to the onset of Type I supernovae, i.e. that star formation in individual halo regions is short-lived (e.g Wyse and Gilmore, 1988), perhaps due to Type II supernova-driven winds as discussed in section 2 above. This does not mean that the global halo star formation phase was short-lived, since the onset of Type I supernovae occurs relative to the initiation of star formation at any particular location, and different parts of halo substructure can initiate star formation at different absolute times (again, as discussed in section 2). However, the iron abundance at which star formation is truncated in halo substructure may well vary, reflecting intrinsic variation in star-formation rates; the absence of stars with element ratios that are consistent with forming in a manner indicated by the dot-dashed line in Figure 1 (for example) means that the gas leftover from halo substructure did not form stars which have been detected at the solar neighbourhood. Proposing that this gas flows towards the Galactic center, to form the bulge, provides an explanation, and is consistent with angular momentum arguments. Removal of gas from the halo has been advocated for years as a means of understanding the low mean enrichment of the halo, assuming an invariant IMF (as the element ratio data now require); gas loss of around ten times the mass remaining in the stellar halo is suggested (Hartwick, 1976), consistent with dynamical analyses of the bulge(/bar). Thus the inhomogeneous model for halo formation that is inherent in CDM-type models requires incorporation of the monolithic collapse and radial infall of Eggen, Lynden-Bell and Sandage 
(1962). Indeed, specific calculation of element ratios in a model where the halo pre-enriches the disk and one has 'contemporaneous evolution of two coupled zones' (e.g. Ferrini et al., 1992) predicts unobserved scatter in the element ratios.

The mean iron abundance of the bulge population was previously estimated to be well above solar metallicity (e.g. Rich, 1988). However, the most recent, high dispersion (echelle) spectroscopy of bulge K giants in Baade's Window (McWilliam and Rich, 1994) has discovered a systematic overestimate in iron abundance in the earlier, lower-resolution, studies, probably due to use of combined $\mathrm{Mg}$ and Fe features, implicitly assuming solar element ratios, and to molecular line blanketing. The overestimate is most severe for the highest chemical abundances, ranging from only about 0.1 $\operatorname{dex}$ at $[\mathrm{Fe} / \mathrm{H}]=-1$ dex to $\sim+0.5$ dex at $[\mathrm{Fe} / \mathrm{H}]=+0.5$ dex. (The ramifications of this for the utility of the bulge as a template for external elliptical galaxies have yet to be fully investigated.) The new results, based on a transformation derived from a sample of ten stars chosen from Rich's sample to cover the range of iron abundance, give a mean iron abundance of -0.3 dex.

Element ratios will also be important discriminants among models of star formation in the bulge, constraining the accumulation time for material at the center and the star formation timescale. If the proto-bulge material accumulated, and formed stars, sufficiently rapidly that only Type II supernovae are available for chemical enrichment, then the element ratios reflect the massive star IMF. Unfortunately the rather sparse elemental abundance data, for only 10 stars (McWilliam and Rich, 1994), are rather confusing, with $\mathrm{Mg}$ and Ti showing enhancements relative to iron indicative of Type II enrichment (with a solar neighbourhood IMF) over the entire range of iron abundances, $\mathrm{Si}$ and $\mathrm{Ca}$, in contrast, having the solar ratio relative to iron for stars more iron-rich than $-0.5 \mathrm{dex}$, and $[\mathrm{O} / \mathrm{Fe}]$ being consistent with solar for all stars. Clearly more data are required.

If the bulge formed 'rapidly', then after star formation has ceased iron will eventually be synthesised in Type I supernova and ejected into the bulge, releasing about the same amount of iron as ejected by Type II supernovae, for a reasonable range of IMFs and models of Type I supernovae (the Type I supernovae from the halo background population that is also present are merely perturbations to the present model, due to the much larger mass, $\sim \times 10$, of the bulge). This gas must be accounted for in the model. Such iron-rich gas, which is not incorporated in the main bulge population, is produced in any model for the bulge which has sufficiently rapid star formation that it is essentially complete prior to Type I explosions. Should this gas sustain a low level of star formation, then one may expect to find extremely iron-rich stars which have very low oxygen abundances 
(cf. Gilmore and Wyse, 1991). The relatively slow, and uncorrelated, rate at which Type I supernovae explode contrasts with Type II supernovae, and it is unlikely that the iron-rich gas will be ejected as a wind, but depending on its density, it may be maintained at a high temperature; the recent detection of X-ray emitting gas at the center may be related (Yamauchi et al., 1991; Blitz et al., 1993).

More direct estimation of the age of the dominant population in the bulge can be attempted by analysis of colour-magnitude diagrams. Fairly model-independently, one may expect the first star formation in the Galaxy to have occurred in the densest regions, which have the shortest dynamical times. The high spatial resolution of HST allows fitting of the mainsequence turn-off, relatively unaffected by crowding. Analysis of both the luminosity function and turnoff of unevolved bulge stars in Baade's window (Holtzman et al., 1993) suggests a typical age of only 5-10 Gyr for a mean metallicity of around half the solar abundance, as the most recent observations imply; an exclusively old bulge is only possible with unlikely combinations of reddening, distance and photometric uncertainties. Extremely well-populated colour-magnitude diagrams of the evolved stars in the bulge have become available as a side-benefit of searches for gravitational micro-lensing events and allow study of shorter-lived (and hence rarer) evolutionary phases. In particular the OGLE collaboration find a substantial population of core-helium-burning 'red clump' stars which they attribute to a large intermediate-age population, significantly younger than 10Gyr (Paczsynski et al., 1994). This is also consistent with the inferences from analysis of the periods of variable stars in the bulge (e.g. Harmon and Gilmore, 1988). However, Renzini (1994) points out that the lifetime of the 'clump' is also sensitive to helium content, and the relative populations of 'clump' and red giant stars may be understandable with an old age but enhanced helium (which obviously depends on the parameter $\Delta Y / \Delta Z$ ). The presence of significantly older stars has been inferred from the horizontal branch morphology, in particular the relative number of RR Lyrae stars, by Lee (1992 - but see also Wyse, 1993). At present the age distribution in the bulge remains rather uncertain.

The presence or otherwise of chemical (and elemental) abundance gradients in the bulge contains clues to bulge formation. A lack of a gradient argues against dissipational formation. The McWilliam and Rich data pertain only to Baade's window, of order 500pc from the Galactic Center. Ibata and Gilmore (1994) have achieved 'long-slit spectroscopy' of the bulge analagous to that done for external galaxies through two series of multi-fibre fields, one parallel to the minor axis, but at $R \sim 3 \mathrm{kpc}$, and one parallel to the major axis, at $Z \sim 3 \mathrm{kpc}$. Their total sample is around 2,000 $\mathrm{K}$ giants. This shows no evidence for gradients, and has a mean metallicity 
(albeit calibrated using a magnesium feature) of $-0.3 \mathrm{dex}$, with a distribution that is remarkably similar to that derived for Baade's window by McWilliam and Rich. Further, the K-giants in the inner disk also have a mean metallicity of -0.3 dex (Lewis and Freeman, 1989). Thus the overall chemical enrichment of the bulge is consistent with a dissipationless formation of the bulge from the inner disk. Such a formation mechanism for the bulge has indeed been proposed - an out-of-plane bending instability in a bar-unstable disk (Combes et al., 1990; Raha et al., 1991). The morphology of the central regions of the galaxy after the bending instability has fattened the bar looks remarkably like the COBE images of our Galaxy, even with a distinct 'peanut' minimum along the minor axis. However, the latest analysis of the DIRBE data (Weiland et al., 1994) concludes that the peanut morphology is an artefact of varying extinction. Also, the scaleheight of the COBE bulge is around 300pc, which is equal to that of the old disk in the solar neighbourhood, despite significant fattening of the bulge relative to the disk being an important feature of the instability. Now, the disk as seen by DIRBE and in the ground-based IR observations is significantly thinner than the local old disk (Kent et al., 1991), but this may be due to the fact that the young disk is preferentially detected - observations of edge-on external galaxies consistently show the (old) disk scale-height to be independent of radius (e.g. van der Kruit and Searle, 1981; Shaw and Gilmore, 1990). Detailed analyses to understand which stellar populations are studied in the IR are needed.

The kinematics of bulge stars - as evidenced in the angular momentum distribution - suggest a closer connection to the halo than to the present disk; detailed predictions for the kinematics of bulge stars in the bending instability model should be made. It may be that the halo ejecta forms the inner disk and still satisfies angular-momentum and element-ratio constraints. It is also noteworthy that there is as yet no compelling evidence from stellar kinematics that the dominant gravitating mass of the bulge region is triaxial; the oblate rotator model (e.g. Kent, 1992) provides an acceptable fit (e.g. Ibata and Gilmore, 1994). Perhaps the firmest indication for triaxiality is provided by the analysis of Zhao, Spergel and Rich (1994) of bulge stars with both proper motions and radial velocities, but this is a small sample with large error bars. The implications for chemical evolution of the inferred triaxial nature of the central regions of the Galaxy (e.g. Blitz and Spergel, 1991; Binney et al., 1991) have not been explored. One obvious effect is the enhanced inflow of gas expected due to the gravitational torques of the bar, perhaps of amplitude $0.01-0.1 \mathrm{M}_{\odot} / \mathrm{yr}$ (Gerhard and Binney, 1993). 


\section{Application to the Disk}

The sample of disk F/G stars of Edvardsson et al. (1993; discussed by Nissen, this volume) has provided an unprecedented opportunity to study the pattern of element ratios for stars for which independent age estimates are available. This allows the star-formation history to be analysed both in terms of relative timescales - relative to the onset of star formation in a particular local star-forming region - and of an absolute timescale in the global scheme of the formation of the Galaxy. Referring back to Figure 1 , stars which formed with a higher star formation rate will sustain the plateau of enhanced $[\mathrm{O} / \mathrm{Fe}]$ to higher $[\mathrm{Fe} / \mathrm{H}]$; this is seen in the Edvardsson et al. sample once stars which are believed (from their kinematics plus a model Galactic potential) to have formed in the inner Galaxy are isolated. Indeed, the data are in good agreement with models that predict the disk formed stars more rapidly in its inner regions, with a smooth variation of star formation rate with radius (e.g. Wyse and Silk, 1989).

The observed lack of scatter in the trend of element ratios, once a subsample formed at a given radius has been isolated, places severe constraints on the variation of the star formation rate and of the IMF (e.g. Wyse and Gilmore, 1988). A monotonically-declining star formation rate is favoured, with a constant IMF.

The Edvardsson et al. sample was defined at the solar neighbourhood, and thus is biased kinematically, so that trends of element ratios with kinematics cannot be easily investigated. However, the element ratios of thickdisk stars appear to match smoothly onto the trend set by the halo stars, with little or no scatter, and as argued above this can only arise within the context of inhomogeneous halo models if the IMF is invariant, and there is no chemical connection between thick disk and halo. This raises the as-yet-unanswered question of what pre-enriches the thick disk, if there is indeed no significant 'metal-poor thick disk', as implied by the current observations which characterise the thick disk as pre-dominantly stars with iron abundance of -0.6dex (see Gilmore, Wyse and Jones, 1995; Ryan and Lambert, 1995).

The lack of scatter in element ratios contrasts with the large scatter in the age-metallicity relationship of this sample; intermediate age stars are seen with essentially the entire range of iron abundance in the sample, from one tenth of solar to above solar. The trend of decreasing age with increasing metallicity is due to essentially only the youngest and oldest stars. It should be noted that stars of approximately half the solar metallicity existed 10Gyr ago, which is relevant to the interpretation of qso absorption-line systems. Also the age determinations of individual thick-disk stars agree with the

more indirect inferences from the metallicity and turn-off $\mathrm{B}-\mathrm{V}$ colour of 
the thick disk, $\sim 15 \mathrm{Gyr}$ (Wyse and Gilmore, 1988). This relatively old age contrasts with that inferred for the disk from analysis of the white dwarf luminosity function (e.g. Winget et al., 1987). Taking both results at face value suggests that there may have been a hiatus in star formation between the earliest disk (now the thick disk) and the main body of the disk. However, an age gap is not seen in the Edvardsson et al. dataset, nor is there any discontinuity in element ratios, as would be expected for a substantial hiatus (see section 3 above).

The scatter in the age-metallicity relationship in the sample of Edvardsson et al. remains even if one restricts the sample to stars one believes formed in a narrow radial range; This suggests that one cannot appeal to a mean abundance gradient (as inherent in models where the inner regions evolve faster, as inferred from the element ratio data) combined with epicyclic orbits to mix stars (but see Fuchs and Weilen's contributed paper this volume). Rather, star formation and chemical evolution are inherently inhomogeneous.

The scatter seen in the age-metallicity relationship plausibly reflects the inhomogeneity of star formation within giant molecular cloud complexes in the disk, provided that star formation regions self-enrich, and GMC complexes dissociate and reform with incomplete mixing of chemical elements. Alternatively, one can appeal to infall of metal-poor gas that forms star prior to complete mixing (White and Audouze, 1980; Edvardsson et al., 1993). Both these scenarios require that different elements mix the same way, despite their different production sites and timescales. Observational input comes from the detailed elemental abundances of main sequence B stars in the four main age subgroups in the Orion association (Cunha and Lambert, 1992). The age difference is $\sim 10^{7} \mathrm{yr}$ across the association, and the youngest subgroup is enhanced in oxygen by 0.2 dex relative to the older subgroups. Although this spread in oxygen seems high, should it be due to enrichment from a typical Type II supernova in the older subgroups, and the iron from that supernova mixed with the oxygen, this produces a trend that is within the limits of scatter set by the element data of Edvardsson et al. at the iron abundance of Orion.

At present no model simultaneously and self-consistently predicts a high amplitude of scatter in the age-metallicity relationship and no detectable scatter in the element ratios. Classical tests of chemical evolution in the thin disk, such as the G-dwarf metallicity distribution which describes the integrated chemical enrichment history of the solar neighbourhood, are predicated on the assumption of a steady increase of metallicity with time, and are difficult to interpret when the dispersion in metallicity at a fixed time is approximately equal to the dispersion when one integrates over time (as pointed out by Tinsley, 1975). Malinie et al. (1993) developed a model 
of inhomogeneous chemical evolution by adopting a priori the amplitude of scatter seen in the dataset of Edvardsson et al. However, they did not attempt self-consistent modelling and to fit the G-dwarf metallicity distribution they were forced as usual simply to assume that the disk was pre-enriched to 0.3 solar abundance (e.g. Pagel and Patchett, 1975; Pagel, 1989b). Gilmore and Wyse (1986) demonstrated that the thick disk, if sufficiently massive, could provide the required self-consistent pre-enrichment of the thin disk. However, that paper appealed to the halo to pre-enrich the thick disk (see also Burkert, Truran and Hensler, 1992). Updated samples of $\mathrm{F} / \mathrm{G}$ stars, with improved metallicity determinations and selection criteria, should provide more detailed testing of solar-neighbourhood chemical evolution (Gilmore and Wyse, 1995; Bikmaev, this volume).

A possible shortcoming of the Malinie et al. treatment of chemical inhomogeneity is that they assume it to be shortlived and erased between episodes of star formation. We (Gilmore and Wyse) are developing models that rather allow inhomogeneities to survive the dispersal and re-formation of the giant molecular cloud complexes in which stars form. This may more accurately reflect the physical processes that operate (e.g. Elmegreen, 1979), particularly in light of the realisation (e.g. Wyse and Silk, 1985; Heiles, 1990; Norman and Ikeuchi, 1989) that the vertical potential of the disk is sufficiently weak that gas from the disk is probably re-cycled through ejection into the halo where it can cool, perhaps without significant mixing.

\section{References}

Barbuy, B. and Erdeyli-Mendes, R., 1989, A. \& A. 214, 239

Binney, J.J., et al., 1991, M. N. R. A. S. 252, 210

Blitz, L. and Spergel, D.N., 1991, Ap. J. 379, 631

Blitz, L. Binney, J., Lo, K.Y., Bally, J. and Ho, P.T.P., 1993, Nature 361, 417

Blumenthal, G.R., Faber, S.M., Primack, J.R. and Rees, M.J., 1984, Nature 311, 517

Burkert, A., Truran, J. and Hensler, G., 1992, Ap. J. 391, 651

Carney, B., 1990, Bulges of Galaxies, eds. B.Jarvis and D.Terndrup, ESO, Garching, p.26

Combes, F., Debbasch, F., Friedl, D. and Pfenniger, D., 1990, A. \& A A. 233, 82

Cunha, K. and Lambert, D., 1992, Ap. J. 399, 586

Dekel, A. and Silk, J., 1986, Ap. J. 303, 39

Edvardsson, B., et al., 1993, A. ES A. 275, 101

Eggen, O.J., Lynden-Bell, D. and Sandage, A.R., 1962, Ap. J. 136, 748

Elmegreen, B., 1979, Ap. J. 231, 372

Fall, S.M. and Efstathiou, G.P., 1980, M. N. R. A. S. 193, 189

Ferrini, F., Matteucci, F., Pardi, C. and Penco, U., 1992, Ap. J. 387, 138

Freeman, K.C., 1991, Dynamics of Disc Galaxies, ed. B. Sundelius, Göteborgs University, Göteborg, p.15

Gerhard, O.E. and Binney, J., 1993, Galactic Bulges, eds. H. Dejonghe and H.J. Habing, Kluwer, Dordrecht, p. 275

Gilmore, G. and Wyse, R.F.G., 1995, submitted to A. J.

Gilmore, G. and Wyse, R.F.G., 1993, First Light in the Universe, 8th IAP Meeting, eds B. Rocca-Volmerange, M. Dennefeld, B. Guiderdoni and J. Tran Than Van, p.177

Gilmore, G. and Wyse, R.F.G., 1991, Ap. J. 367, L55 
Gilmore, G. and Wyse, R.F.G., 1986, Nature 322, 806

Gilmore, G., Wyse, R.F.G. and Jones, J.B., 1995, A. J., in press

Gilmore, G., Wyse, R.F.G. and Kuijken, K., 1989, Ann. Rev. Astron. Astroph. 27, 555

Gunn, J.E., 1987, The Galaxy, eds. G. Gilmore and B. Carswell, Reidel, Dordrecht, p.413

Harmon, R.A. and Gilmore, G., 1988, M. N. R. A. S. 235, 1025

Heiles, C., 1990, Ap. J. 354, 483

Holtzman, J.A., et al., 1993, A. J. 106, 1826

Ibata, R., Gilmore, G., and Irwin, M., 1994, Nature 370, 194

Hartwick, F.D.A., 1976, Ap. J. 209, 418

Iben, I. and Tutukov, A.V., 1987, Ap. J. 313, 727

Kent, S.M., 1992, Ap. J. 387, 181

Kent, S.M., Dame, T.M. and Fazio, G., 1991, Ap. J. 378, 131

Kruit, P.C. van der and Searle, L., 1981, A. \& A. 95, 105

Lacey, C.G., 1991, Dynamics of Disc Galaxies, ed. B. Sundelius, Göteborgs University, Göteborg, p.257

Lacey, C.G. and Cole, S., 1993, M. N. R. A. S. 262, 627

Lee, Y.-W., 1992, A. J. 104, 1780

Lewis, J. and Freeman, K.C., 1989, A. J. 97, 139

Malinie, G., Hartmann, D.H., Clayton, D.D. and Mathews, G.J., 1993, Ap. J. 413, 633

Matteucci, F. and Greggio, L., 1986, A. E A. 154, 279

McWilliam, A. and Rich, M., 1994, Ap. J. Suppl. 91, 749

Navarro, J., Frenk, C.S. and White, S.D.M., 1994, M. N. R. A. S. 267, L1

Nissen, P.E., Gustafsson, B., Edvardsson, B. and Gilmore, G., 1994, A. \& A., in press

Norman, C.A. and Ikeuchi, S., 1989, Ap. J. 345, 372

Ostriker, J.P., 1990, Evolution of the Universe of Galaxies, ed. R.G. Kron, A.S.P. Conf. Series 10, A.S.P., San Francisco, p.25

Paczynski, B., et al., 1994, A. J. 107, 2060

Pagel, B.E.J., 1994, IAC Winter School on Galaxy Formation and Evolution, ed. C. Munoz-Turon, CUP, Cambridge, in press

Pagel, B.E.J., 1989a, Rev. Mex. Astr. Astrofis. 18, 153

Pagel, B.E.J., 1989b, Evolutionary Phenomena in Galaxies, eds. J. Beckman and B.E.J. Pagel, CUP, Cambridge, p.201

Pagel, B.E.J. and Patchett, B.E., 1975, M. N. R. A. S. 172, 13

Quinn, P.J., Hernquist, L. and Fullager, D.P., 1993, Ap. J. 403, 74

Quinn, T. and Binney, J.J., 1992, M. N. R. A. S. 255, 729

Raha, A., Sellwood, J., James, R. and Kahn, F.D., 1991, Nature 352, 411

Rana, N.C., 1991, Ann. Rev. Astron. Astroph. 29, 129

Renzini, A., 1994, A. \& A. 285, L5

Rich, R.M., 1988, A. J. 95, 828

Ryan, S.J. and Lambert, D.A., 1995, A. J., in press

Ryden, B.S., 1988, Ap. J. 329, 589

Searle, L. and Zinn, R., 1978, Ap. J. 225, 357

Shaw, M.A. and Gilmore, G., 1990, M. N. R. A. S. 242, 59

Silk, J. and Wyse, R.F.G., 1993, Phys. Rep. 231, 293

Smecker, T.A. and Wyse, R.F.G., 1991, Ap. J. 372, 448

Smecker-Hane, T.A. and Wyse, R.F.G., 1992, A. J. 103, 1621

Tinsley, B.M., 1975, Ap. J. 197, 159

Tinsley, B.M., 1976, Ap. J. 208, 797

Tinsley, B.M., 1979, Ap. J. 229, 1046

Tosi, M., 1988, A. \& A. 197, 33

Toomre, A., 1977, The Evolution of Galaxies and Stellar Populations, eds. B.M. Tinsley and R.B. Larson, Yale Univ. Obs, New Haven, p.401

Truran, J.W., 1987, 13th Texas Symposium on Relativistic Astrophysics, ed. M.P. Ulmer, Singapore, World Scientific, p.430

Weiland, J.L. et al., 1994, Ap. J. 425, L81 
Wheeler, J.C., Sneden, C. and Truran, J.W., 1989, Ann. Rev. Astron. Astroph. 27, 279

White, S.D.M and Audouze, J., 1983, M. N. R. A. S. 203, 603

Winget, D.E. et al., 1987, Ap. J. 315, L77

Wyse, R.F.G., 1993, Nature 361, 204

Wyse, R.F.G. and Gilmore, G., 1988, A. J. 95, 1404

Wyse, R.F.G. and Gilmore, G., 1992, A. J. 104, 144

Wyse, R.F.G. and Silk, J., 1985, Ap. J. 296, L1

Wyse, R.F.G. and Silk, J., 1989, Ap. J. 339, 700

Yamauchi, Y. et al., 1991, Ap. J. 365, 532

Zhao, H., Spergel, D.N. and Rich, R.M., 1994, preprint

Zurek, W., Quinn, P. and Salmon, J., 1988, Ap. J. 330, 519 